\title{
Searches at LEP2 Latest Results
}

\author{
M. Begalli \\ Instituto de Física, Universidade do Estado do Rio de Janeiro, UERJ \\ Rua SøFrancisco Xavier, 524/3023-D, 20559-900, Rio de Janeiro, RJ, Brasil \\ (member of the DELPHI Collaboration)
}

Received on 30 March, 2001

\begin{abstract}
Searches for the Higgs boson of the Standard Model and supersymmetric models have been performed by the four LEP experiments in the data collected over almost 12 years (August/1989 until November/2000) at centre-of-mass energies varying from $91.2 \mathrm{GeV}$ to $209 \mathrm{GeV}$. An excess of candidates for the process $e^{+} e^{-} \rightarrow Z^{*} \rightarrow Z^{0} H^{0}$ was found for Higgs masses near $114 \mathrm{GeV}$.
\end{abstract}

\section{Introduction}

The LEP (Large Electron Positron) accelerator, placed at CERN, the European Laboratory for Particle Physics, has succesfully delivered $e^{+} e^{-}$colisions with energies up to $209 \mathrm{GeV}$ for almost 12 years.

The LEP collider's initial energy was chosen to be around $91 \mathrm{GeV}$, so that in these collisions a $Z^{0}$ particle would be produced. The theory of fundamental particles called the Standard Model has been critically tested by studying the creation and decay of the $Z^{0}$. These studies still continue. The $Z^{0}$ is very short-lived, so its presence has to be inferred from its disintegration fragments, which may vary from two to nearly a hundred. Since the end of 1995, LEP has moved on from the $Z^{0}$ and entered its second phase. Its energy was doubled to allow the study of the production of the $Z^{0} Z^{0}$ pairs and of $W^{+} W^{-}$pairs, the charged counterparts of the $Z^{0}$, thus opening a new domain of investigations and Standard Model tests as well as the search for new particles, particularly the Higgs boson and/or supersymmetric particles.

\section{The experiments}

Four experiments were placed in the LEP ring, namely ALEPH, DELPHI, L3 and OPAL. They are general purpose experiments. They have a silicon vertex detector capable to reconstruct the decay of short lived particles like the ones formed by the $\mathbf{b}$ quarks, a tracking chamber in order to reconstruct the trajectory of all charged particles produced in the $e^{+} e^{-}$collisions, electromagnetic and hadronic calorimeters to measure the energy of the electrons, photons and hadrons, and muon chambers. Details of each experiment can be found in [1]. Fig.1 shows a picture of the DELPHI experiment as an example.
Since August 1989, when they started the data taking, until November 2000, when LEP was stopped, an integrated luminosity of $2.5 \mathrm{fb}^{-1}$ was collected.

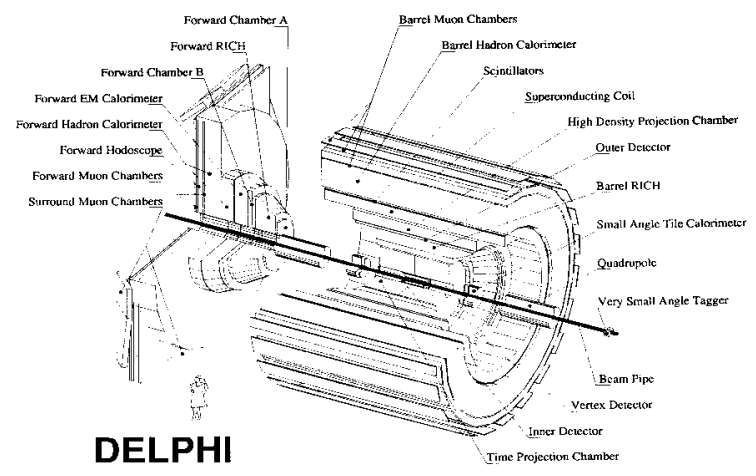

Figure 1. The DELPHI experiment at LEP.

Luminosity is a very usefull number in high energy physics. The number of events produced in a certain process is given by

$$
N=L \sigma
$$

where $N$ is the number of events, $\sigma$ is the cross section of the given process and $L$ is the luminosity. To measure the Luminosity a well known process is used: the Bhabha scattering, $e^{+} e^{-} \rightarrow e^{+} e^{-}$. Calorimeters are placed very forward in each of the detectors in order to register both the electron and the positron from these interactions. Since the momentum of the electron (and the positron) is much higher than its mass the differential cross section in the centre-of-mass system is calculated taking into account many orders in perturbation theory, bringing us to a very accurate value. Table 1 shows the luminosity per centre-of-mass energy per year 
per LEP experiment. One has to notice that although the luminosity is practically the same for the $Z^{0}$ mass peak and for the data taken during year 2000, the first one means a much higher number of event since we were at a peak of the cross section for the process $e^{+} e^{-}$.

From these values we are able to predict the number of expected events for any process we want to study.

\section{Searches}

The searches at LEP experiments are performed in a very broad way. We try to look everywhere, consider all the models and investigate each channel and each fluctuation. In order to get the highest available number of events (or luminosity) the 4 experiments combine the results, discuss all the anomalies found by an experiment and cross-check the analysis getting a higher chance to find a new particle if it was produced at LEP.

Direct searches are done in the framework of a specific model (supersymmetry or charged Higgs, for example) looking for the expected signal. Excesses in any final state topology are also studied since they will trigger new models. Indirect searches take the Standard Model as the null hypothesis and see if there are deviations.

In this paper we will present the search for the Standard Model Higgs and for the Higgses predicted by the MSSM (Minimal Super Symmetric Model). All results are taken from the presentations at the LEPC (LEP Commitee)[2], from the publications of the 4 experiments[3] and references therein.

\section{The Standard Model Higgs}

The theory of the Standard Model, SU(2) $\times \mathrm{U}(1)$, describes at the per mill level the couplings of quarks and leptons to $\gamma, Z^{0}, W^{ \pm}$and (to less accuracy) the triple gauge vertices: the gauge symmetry is indeed exact. Yet the particles are note degenerate in mass i.e. the symmetry is broken in the masses: the symmetry is spontaneously broken via the Higgs mechanism.

The existence of the Higgs boson is well supported by the data on radiactive correction but, which is the value of its mass?

Precision tests of the Standard Model have recently been performed by LEP, SLD and the Tevatron, at FERMILAB (USA). In the data there was no significant evidence for departure from the Standard Model with precision of the order of $0.1 \%([5])$, as can be seen in Fig. 2. Fig. 3 shows the $\chi^{2}$ fit for the combination of all those results for a Higgs mass varying between 10 and $1000 \mathrm{GeV} / \mathrm{c}^{2}$. The theory uncertainty is shown as well as the results for two different values of $\Delta \alpha_{\text {hadrons }}$. The mass value already excluded by the directed searches performed at LEP is $113,5 \mathrm{GeV}$. The fit results give a lower limit for the Higgs mass of $m(H)=62_{-30}^{+53} \mathrm{GeV} / \mathrm{c}^{2}$ and an upper limit of $m(H)=170 \mathrm{GeV} / \mathrm{c}^{2}$ at $95 \%$ Confidence Level.

The Higgs boson can be produced at LEP via Higgsstrahlung combined with gauge boson fusion. Fig. 4 shows the diagrams for these processes. The plot for the Higgs production cross section as a function of its mass is given in Fig.5. It is clear that at LEP energies the Higgsstrahlung plays the major contribution since our mass reach is $115 \mathrm{GeV} / \mathrm{c}^{2}$. For such values of mass the Higgs predominantly decay into $\mathbf{b}$ quarks, $B R\left(H^{0} \rightarrow b \bar{b}\right) \approx 85 \%$. The second most probable decay channel is the $B R\left(H^{0} \rightarrow \tau^{+} \tau^{-}\right) \approx 8 \%$. For data taken above $200 \mathrm{GeV}$ the channels $H^{0} \rightarrow W^{+} W^{-}$and $H^{0} \rightarrow g g$ were also studied.

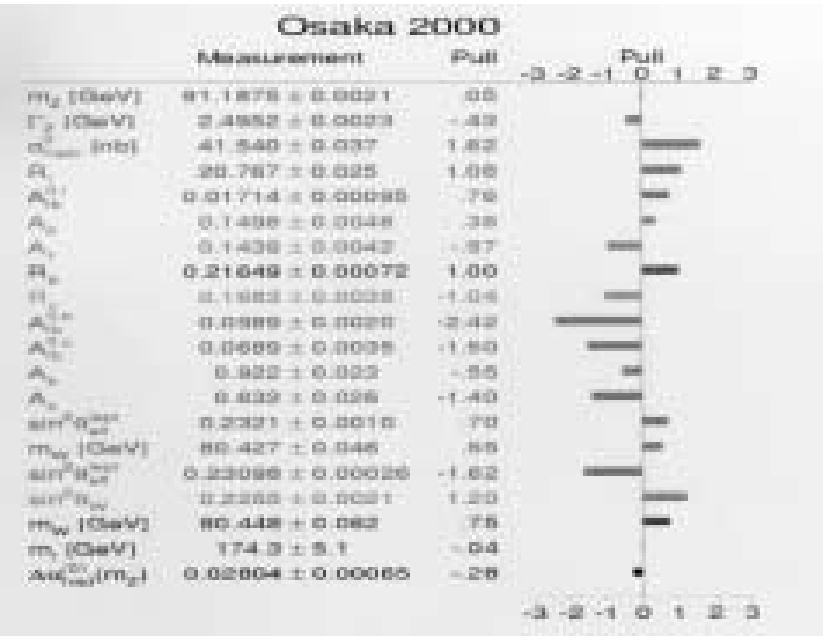

Figure 2. Precision tests of the Standard Model.

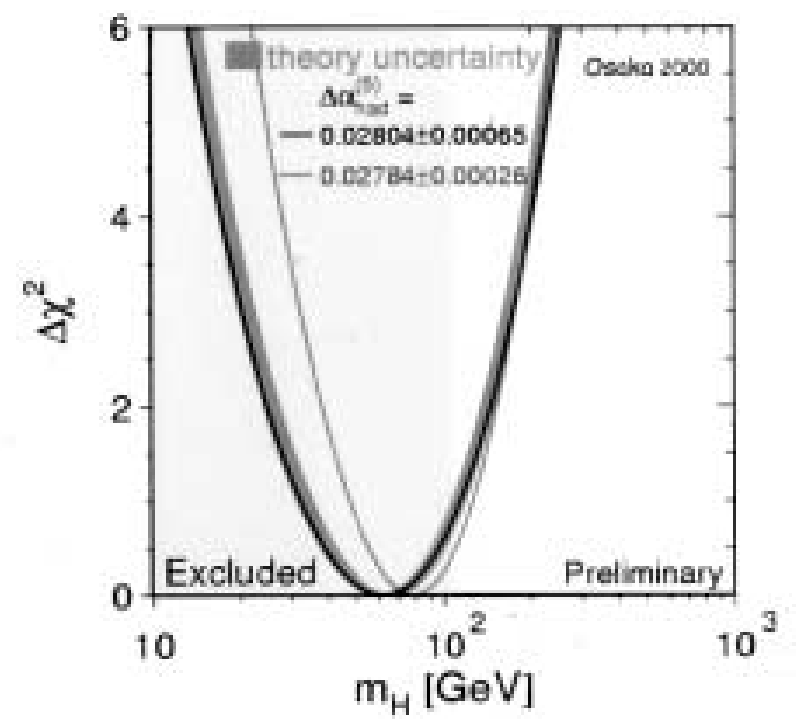

Figure 3. The $\chi^{2}$ fit for all the tests in the Standard Model for a Higgs mass varying between 10 and $1000 \mathrm{GeV} / \mathrm{c}^{2}$.

If the Higgs was produced via $e^{+} e^{-} \rightarrow Z^{*} \rightarrow H^{0} Z^{0}$ we expect to have 3 topologies for an event having a Higgs candidate (see Fig. 6) 
- 4 jets: 2 jets coming from the $Z^{0} \rightarrow q \bar{q}$ decay and the other 2 from the Higgs decay in a pair of b quarks in the event. Each jet comes from the hadronization of each of the quarks. Such topology is the most probable one, it occurs $60 \%$ of the time.

- 2 jets and missing energy: the missing energy is due to the decay $Z^{0} \rightarrow \nu \bar{\nu}$ and the 2 jets from the Higgs decay. The probability here is $19 \%$.

- 2 jets and 2 leptons: here $Z^{0} \rightarrow e^{+} e^{-}, \mu^{+} \mu^{-}$giving a very clear signature, the Higgs produces the 2 jets. Unfortunately it is the less probable case, only $6 \%$.

When the Higgs decays in a pair of $\tau$ leptons the topologies remains the same, only the jets coming from its decays have very low multiplicity $(1,3$ or 5 charged tracks).

Many channels can have the same topology as a Higgs event. In order to reduce the background, which is some orders of magnitude higher than the signal, a set of cuts was applied by each experiment to the reconstructed events.

- $e^{+} e^{-} \rightarrow q \bar{q}$ and $e^{+} e^{-} \rightarrow Z^{0} \gamma$ are the dominant background but easy to reduce once we take into account the hermeticity of the detectors.

- $e^{+} e^{-} \rightarrow e^{+} e^{-} Z^{0}$ and $e^{+} e^{-} \rightarrow e^{ \pm} \nu W^{\mp}$ is reduced by analysing the missing momentum and the missing energy of the event.

- special care is needed with 4 -fermion, $e^{+} e^{-} \rightarrow$ $Z^{0} Z^{0}$ and $e^{+} e^{-} \rightarrow Z^{0} g g$ events, where $g$ stands for gluon. In this case, a good mass reconstruction is needed both for the $Z^{0}$ and for the Higgs.

- $e^{+} e^{-} \rightarrow W^{+} W^{-}$is an irreducible background for the studies of the charged Higgs production, $e^{+} e^{-} \rightarrow H^{+} H^{-}$.

- $e^{+} e^{-} \rightarrow Z^{0} Z^{0}$ is an irreducible background for the Standard Model and the supersymmetric Higgs search, namely $e^{+} e^{-} \rightarrow Z^{0} H^{0}$ and $e^{+} e^{-} \rightarrow h^{0} A^{0}$.
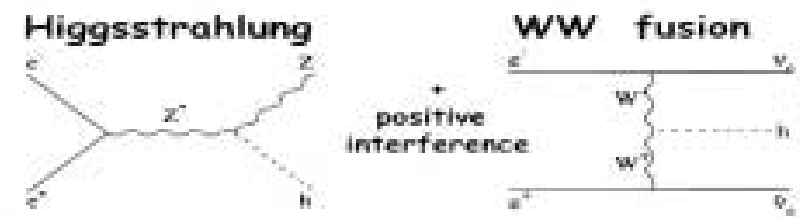

Figure 4. The diagrams for the production of the Higgs boson at LEP: Higgsstrahlung combined with gauge boson fusion.

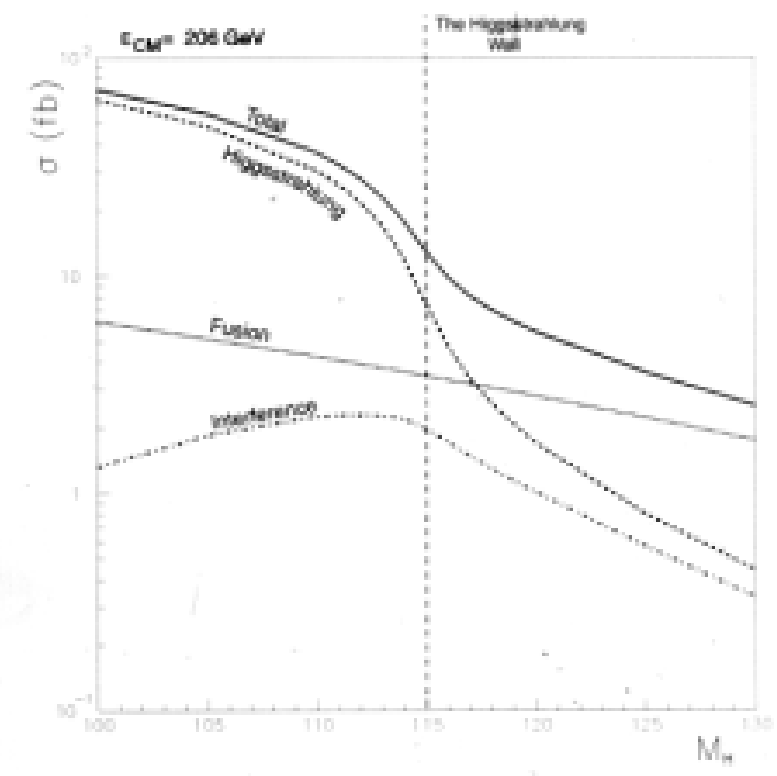

Figure 5. Cross section for Higgs boson production in $e^{+} e^{-}$ collisions as a function of its mass.

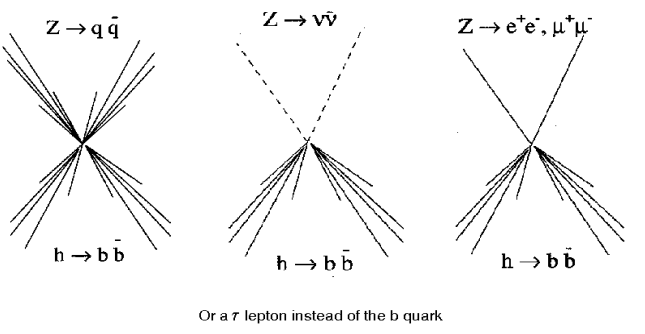

Figure 6. The different topologies of the events $e^{+} e^{-} \rightarrow$ $Z^{0} H^{0}$.

The $b$-tagging is the fundamental tool to reduce the background. Combining a set of selection cuts, which includes a well reconstructed secondary vertex compatible with the $b$-lifetime, it gives a very accurate identification of the $b$-decays. Fig. 7 shows the distributions of the variables used in the $b$-tagging by DELPHI, namely the lifetime, the mass of the secondary vertex, the fraction of the charged energy, the missing transverse momentum of the secondary vertex, the lepton identification and transverse momentum in case of a semi-leptonic decay and the track rapidity. The agreement obtained between real data (RD) and the Monte Carlo (MC)is also shown. Fig. 10 presents a close-up of the vertex region of a double $b$-tagged event, a Higgs candidate, found by L3.

To live with the remaining background one has to search for an excess in the mass distribution for the Higgs candidates. Fig. 8 shows the reconstructed Higgs mass distribution for a loose, medium and tight selection in the searches performed in the 4 jets topology. 
They differ by asking a different value for the relation signal/background (S/B). Since it is impossible to eliminate completely the background, a neural network and/or a discriminant likelihood analysis was used by the 4 experiments in order to get a better calculation of the probability of an event to be signal or background. Therefore the first plot in Fig. 4 was done by requiring $S / B=0.3$, the second plot by requiring $S / B=1.0$ and the third $S / B=2.0$ at least. It means, an event will never be called a Higgs event but a candidate with high probability to be a Higgs, if this will be the case. One can see that it is possible to observe an excess of the signal over the background if the mass of the Higgs boson is of the order of $115 \mathrm{GeV} / \mathrm{c}^{2}$.

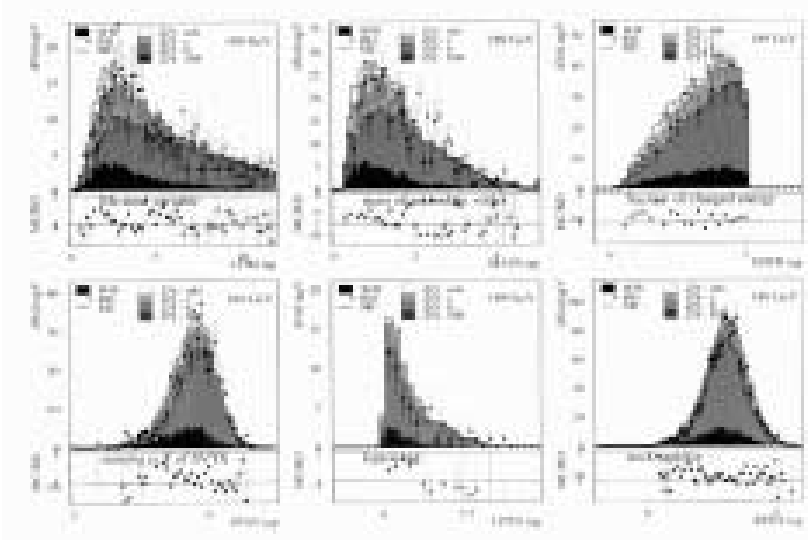

Figure 7. The distributions of the variables used in the $b$ tagging by DELPHI.

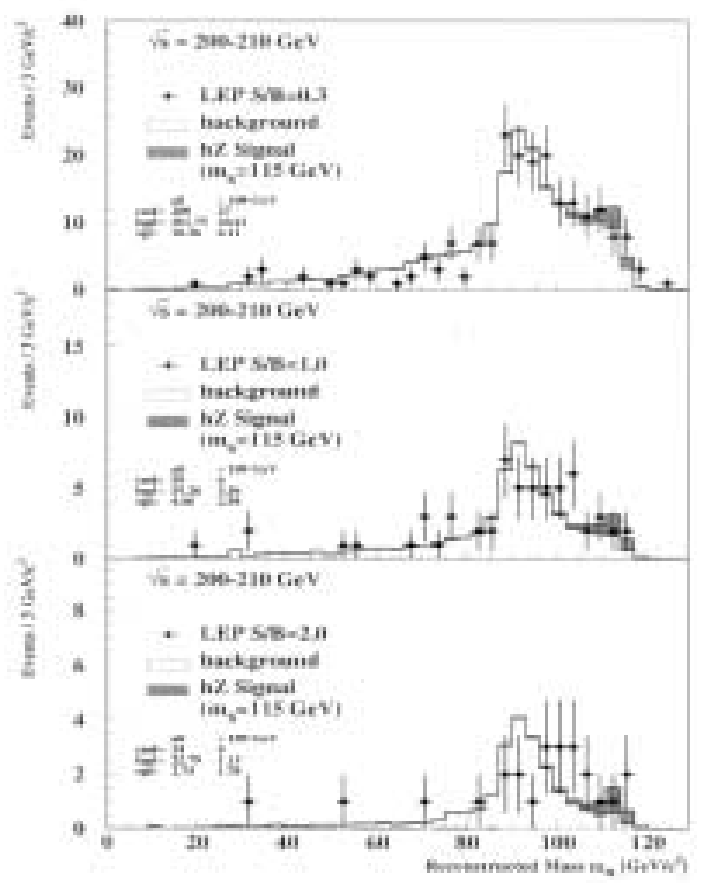

Figure 8. Distributions of reconstructed mass for the loose $(\mathrm{S} / \mathrm{B}=0.3)$, medium $(\mathrm{S} / \mathrm{B}=1.0)$ and tight $(\mathrm{S} / \mathrm{B}=2.0)$ selection.

\section{A. 2 jets and 2 leptons channel}

As it was said before, the topology with 2 leptons and $2 b$-quarks is the cleanest one to be analysed. In this case, the 2 leptons must be well identified as electron, muon or tau, be well isolated from the jets (in order to avoid the background from $e^{+} e^{-} \rightarrow b \bar{b}(\gamma)$ and $e^{+} e^{-} \rightarrow c \bar{c}(\gamma)$ events where both quarks decay semileptonicaly), fit a $Z^{0}$ mass and have 2 jets tagged as $\mathbf{b}$. Special care is taken for the case with a pair of taus, because of the neutrinos originated in the tau lepton decay. A good agreement between the data and the expected background was found by the 4 experiments, showing no evidence for a Higgs candidate in this topology.

\section{B. 2 jets and missing energy channel}

The main characteristics of these events is the missing energy of the order of the $Z^{0}$ mass. The $b$-jets must be acoplanar, the missing momentum should never point to a crack in the detector and a special care with the $Z^{0} Z^{0}$ production and the symmetric double radiative return must be taken. In the double $Z^{0}$ production one of the bosons can decay in 2 neutrinos faking a candidate event. The process $e^{+} e^{-} \rightarrow Z^{0} \gamma \gamma$ is an irreducible background. DELPHI has measured the cross section for it and, as can be seen in Fig. 9, only few of those events have both photons reconstructed. In both cases a good measurement of the tracks and of the energy in the calorimeters is necessary in order to obtain a good mass reconstruction of the jets. A recoil fit to the $Z^{0}$ mass is always performed in order to cut off the $Z^{0} Z^{0}$ events.

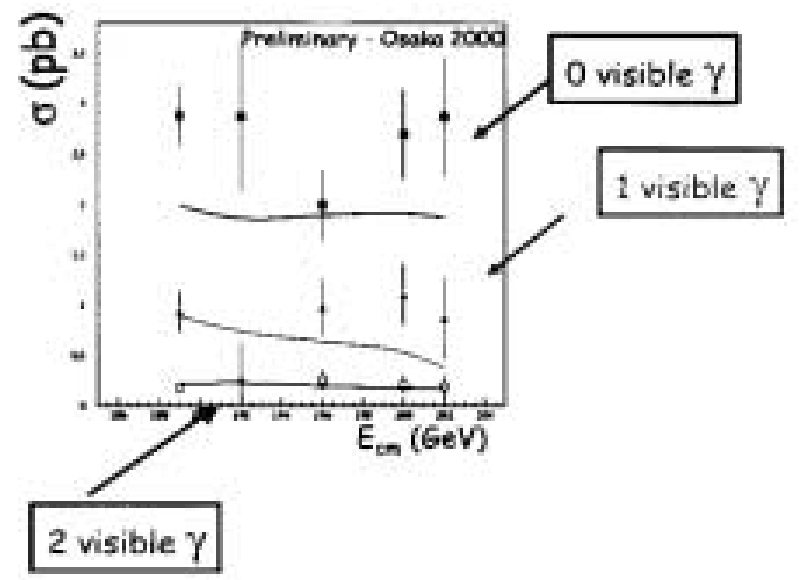

Figure 9. Cross sections as a function of the center of mass energy measured by DELPHI for the process $e^{+} e^{-} \rightarrow Z^{0} \gamma \gamma$.

L3 found 1 candidate event in this topology where 0.16 background and 0.38 signal events were expected. This event, shown in Fig.10, presents two nearly backto-back jets with a large amount of missing energy and 
very little missing momentum, compatible with the production of the Higgs and the $Z^{0}$ nearly at rest. The visible mass is $111 \mathrm{GeV} / \mathrm{c}^{2}$. Assuming a $Z^{0}$ boson recoiling against the Higgs, the fitted mass is $114.4 \mathrm{GeV} / \mathrm{c}^{2}$ with a resolution of $3 \mathrm{GeV} / \mathrm{c}^{2}$. The event has a high $b$-tag value (probability). One jet has a very clear secondary vertex $7.3 \mathrm{~mm}$ from the primary, with a large visible mass.

\section{measured $\mathrm{H}$ mass $=114.4 \mathrm{GeV}$ $\mathrm{H}$ mass resolution $\sim 3 \mathrm{GeV}$}

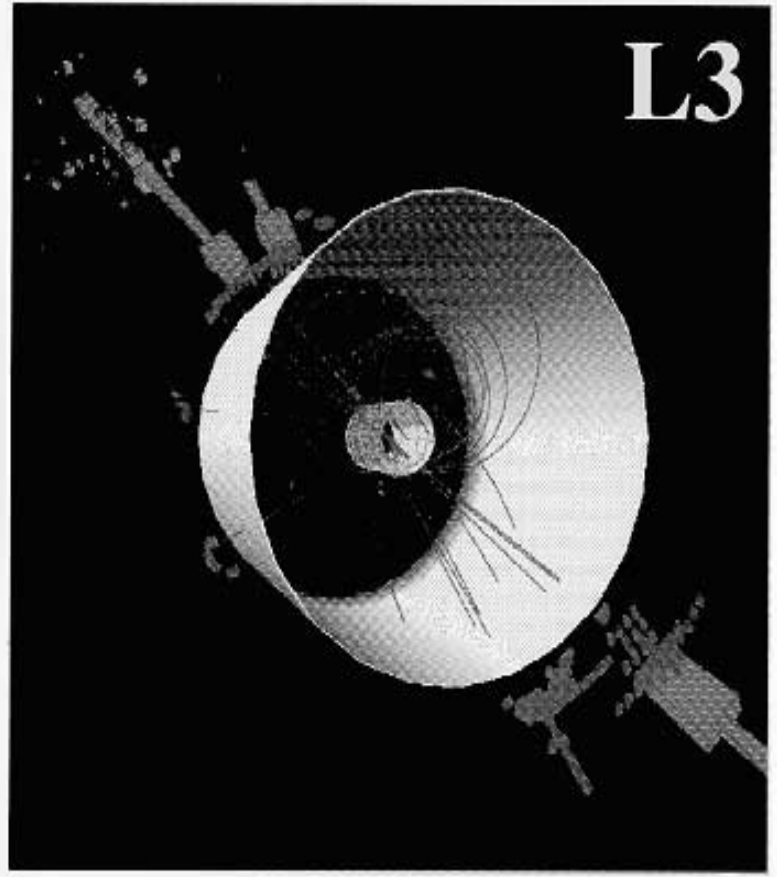

Secondary vtx's view

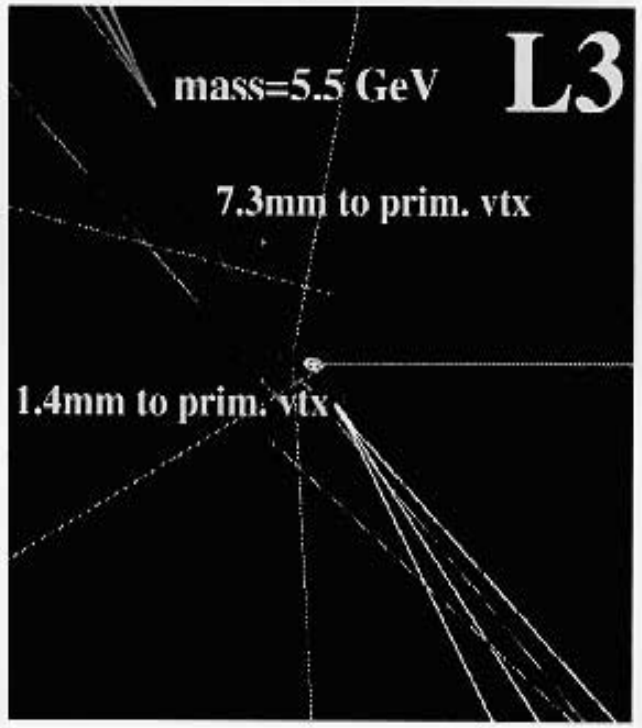

Figure 10. The most significant $H^{0} \nu \nu$ candidate found by L3 with a close-up view of the vertex region.

\section{4 jets channel}

Events are selected out of the hadronic sample by forcing them to have 4 jets and asking at least one of them to be $b$-tagged. This already reduces the $q \bar{q}(\gamma)$, two photon interaction and the $Z^{0} \gamma^{*}$ background. Using the DURHAM algorithm the jets are again reconstructed and the $b$-tagging is applyed giving a global value defined as the maximum $b$-tag value for any dijet in the event, computed as the sum of the corresponding jet $b$-tagging values. A Neural Network (NN) combining different number of variables (it depends on the experiment, DELPHI, for example, uses 13) is used to further select the events. The first variable for the $\mathrm{NN}$ is always the global $b$-tagging value of the event.

Test of the kinematical compatibility of the event with the hypotheses of $W^{+} W^{-}$and $Z^{0} Z^{0}$ pairproduction are performed in either 4 or 5 jet configurations. First, constrained fits are used to derive the probability density function measuring the compatibility of the event kinematics with the production of two objects of any masses. This two-dimensional probability, the ideogram probability is then folded with the expected mass distribution for both pair-production processes.

Further cuts are performed by each experiment depending on their particular analysis. The choice of the Higgs dijet makes use of both the kinematical $5 \mathrm{C}$-fit probabilities and the $b$-tagging information in the event. The likelihood pairing function,

$$
\begin{array}{r}
\left(\left(1-P_{b}^{Z}-P_{c}^{Z}\right) * P_{b}^{j_{1}} * P_{b}^{j_{2}} * P_{q}^{j_{3}} * P_{q}^{j_{4}}+P_{b}^{Z}\right) \\
\left.* P_{b}^{j_{1}} * P_{b}^{j_{2}} * P_{b}^{j_{3}} * P_{b}^{j_{4}}+P_{c}^{Z}\right) \\
\left.* P_{b}^{j_{1}} * P_{b}^{j_{2}} * P_{c}^{j_{3}} * P_{c}^{j_{4}}\right) * P_{3,4}^{5 C}
\end{array}
$$

is calculated for each of the six possibilities to combine the jets $j_{1}, j_{2}, j_{3}, j_{4}$ with $P_{b}^{j_{i}}, P_{c}^{j_{i}}, P_{q}^{j_{i}}$ being the probability of getting the observed $b$-tagging value for the jet $j_{i}$ when originating from a $b, c$ or light quarks, estimated from simulation. $P_{b}^{Z}$ and $P_{c}^{Z}$ are the hadronic branching ratios of the $Z^{0}$ into $b, c$ quarks and $P_{3.4}^{5 C}$ is the probability corresponding to the kinematical 5C-fit with the jets $j_{3}$ and $j_{4}$ assigned to the $Z^{0}$. Selecting the pairing combination that maximizes this funcion, the proportion of right matchings for the Higgs di-jet, estimated in simulated signal events with 114 $\mathrm{GeV} / \mathrm{c}^{2}$ is above $70 \%$ at the tight level, keeping a low rate of wrong, cross-legged, pairings for $Z^{0} Z^{0}$ background events.

Three candidates were found by ALEPH. The first high purity candidate, shown in Fig.11, at a centreof-mass energy of $206.6 \mathrm{GeV}$ was reconstructed with a Higgs boson mass of $110.0 \mathrm{GeV} / \mathrm{c}^{2}$. Three of the four jets are well $b$-tagged and the event was selected as a $4 b$ event with the sum of the four $b$-tagging neural network output values equal to 3.05 . The lowest $b$-tagged 
jet with a value of 0.214 is selected as one of the Higgs boson jets. The probability for any jet in a $4 b$ event to have such a low $b$-tagging value is $19 \%$.

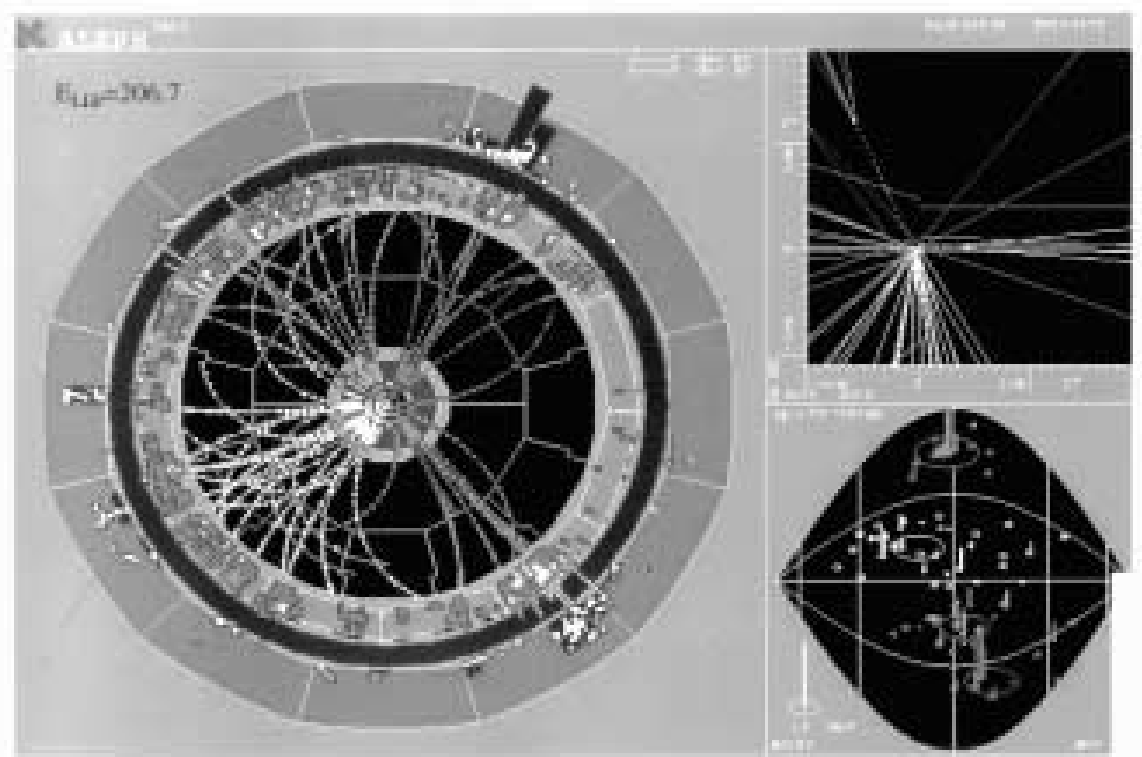

Figure 11. Four-jet Higgs boson candidate with a reconstructed Higgs boson mass of $110.0 \mathrm{GeV} / \mathrm{c}^{2}$. Three of the four jets are well $\mathrm{b}$ tagged. The event is shown in the view transverse to the beam direction and in a closeup of the charged particles in the vertex region.

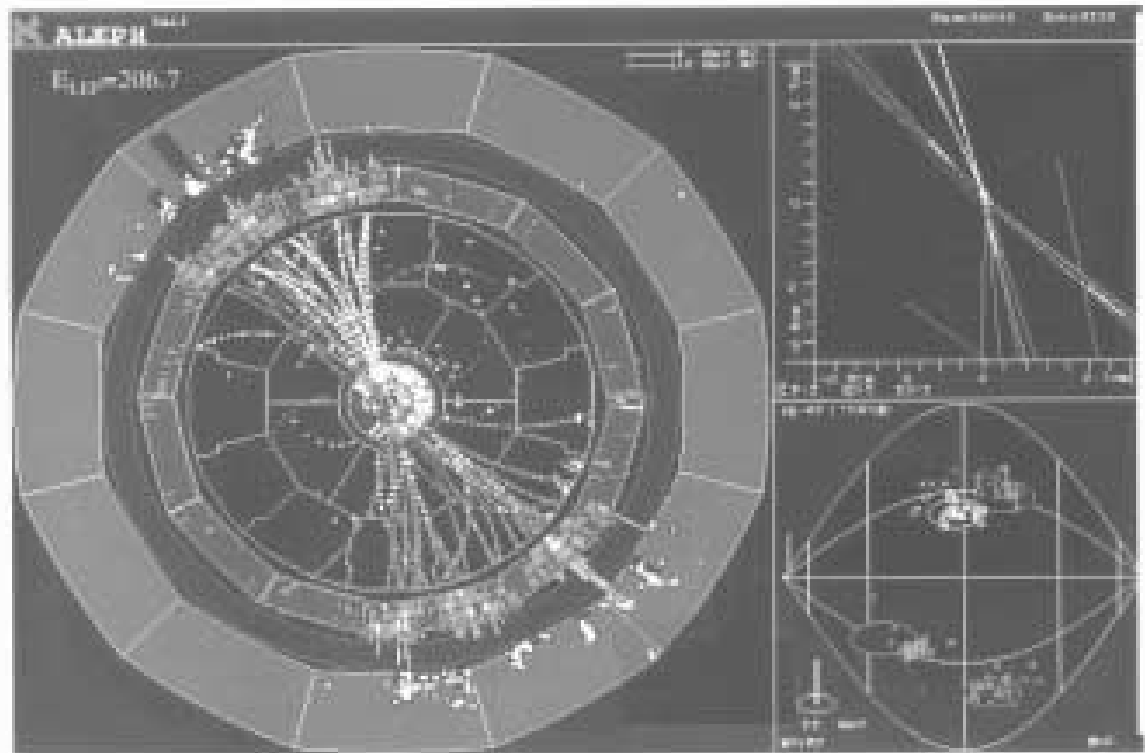

Figure 12. Four-jet Higgs boson candidate with a reconstructed Higgs boson mass of $112.9 \mathrm{GeV} / \mathrm{c}^{2}$. All four jets are well $\mathrm{b}$ tagged. The event is shown in the view transverse to the beam direction and in a closeup of the charged particles in the vertex region. 


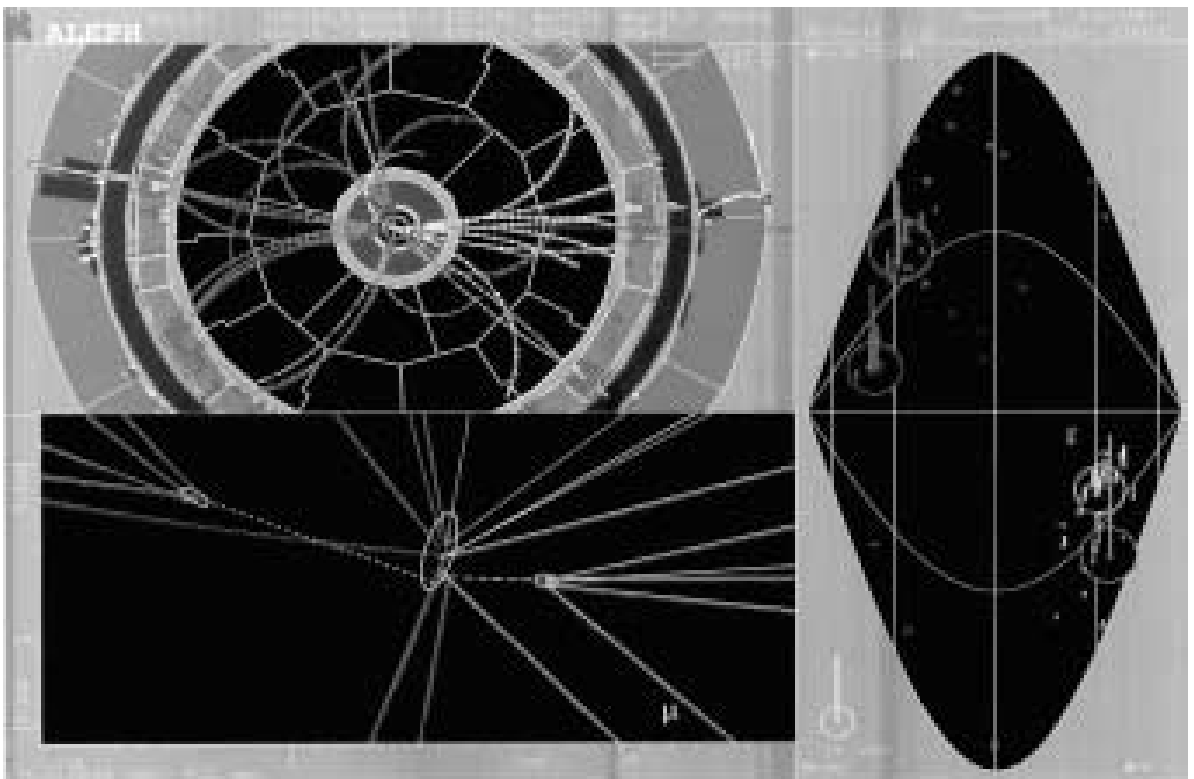

Figure 13. Four-jet Higgs boson candidate with a reconstructed Higgs boson mass of $112.9 \mathrm{GeV} / \mathrm{c}^{2}$. All four jets are well $\mathrm{b}$ tagged. The event is shown in the view transverse to the beam direction and in a closeup of the charged particles in the vertex region.

As the event is identified as a $4 b$ event, any of the six pairing combinations can be considered in its interpretation. The pairing most compatible with the $Z^{0} Z^{0}$ hypothesis, using a fit including the $Z^{0}$ boson width and mass resolutions, gives large fitted $Z^{0}$ boson masses of $98.9 \mathrm{GeV} / \mathrm{c}^{2}$ and $101.6 \mathrm{GeV} / \mathrm{c}^{2}$.

The second high purity candidate, shown in Fig.12, has a reconstructed Higgs boson mass of $112.9 \mathrm{GeV} / \mathrm{c}^{2}$. All four jets in the event are well $b$-tagged with a $b$ tagging neural network sum of 3.76. The measured visible energy in this event is $252 \mathrm{GeV}$, which is much larger than that allowed by the energy resolution of about 10 $\mathrm{GeV}$ for an event with a centre-of-mass energy of 206.7 $\mathrm{GeV}$. A $22 \mathrm{GeV}$ electromagnetic shower was detected in the small angle calorimeter (SICAL) in the plane of the accelerator. As there is too much reconstructed energy and the momentum imbalance is in the opposite direction to the $22 \mathrm{GeV}$ energy deposit, this shower is most likely a beam-related particle, unrelated to the rest of the event. Although the overlapping of such beam-related background is not frequent, the $22 \mathrm{GeV}$ of energy is consistent with the observation in events triggered at random beam crossings.

If this low angle energy deposit is removed from the event, the reconstructed Higgs boson mass increases from $112.9 \mathrm{GeV} / \mathrm{c}^{2}$ to $114.5 \mathrm{GeV} / \mathrm{c}^{2}$. The neural network for this event is stable and changes from 0.997 to 0.998. As the reconstructed Higgs boson mass shifts close to the excess, the significance of the excess would increase by $0.2 \sigma$.

Even if the $22 \mathrm{GeV}$ particle is removed from the event, there is still an energy excess of $23.3 \mathrm{GeV}$ indicating a mismeasurement of jet energy. Such mismeasurement is often due to fake neutral hadrons, i.e., hadronic showers which should have been assigned to a charged particle. This causes double couting in the computation of the energy of the jet. Indeed, a detailed inspection of one of the jets shows that a $13 \mathrm{GeV}$ neutral hadron is likely to have been misidentified. If this object is removed from the jet and the Higgs boson mass recomputed, excluding at the same time the low angle (SICAL) object, a value of $114.2 \mathrm{GeV} / \mathrm{c}^{2}$ is obtained. The very small variation in the reconstructed Higgs boson mass occurs because the fitted masses depend more strongly on the measured jet directions than on the jet energies.

As for the first candidate, the best background explanation for this event is the $Z^{0} Z^{0}$ hypothesis with a different jet pairing. The $Z^{0}$ boson masses from a fit for the most probable $Z^{0} Z^{0}$ pairing choice are $94.0 \mathrm{GeV} / \mathrm{c}^{2}$ and $97.3 \mathrm{GeV} / \mathrm{c}^{2}$.

The third high purity four-jet candidate, shown in Fig.13, at a centre-of-mass energy of $206.7 \mathrm{GeV} / \mathrm{c}^{2}$, has a reconstructed Higgs boson mass of $114.3 \mathrm{GeV} / \mathrm{c}^{2}$. Both of the Higgs boson jets are very well $b$-tagged with well measured displaced vertices and neural network values of 0.999 . The $13.8 \mathrm{GeV} / \mathrm{c}$ missing momentum in the event points to the middle of the Higgs boson jet containing an identified muon coming from the secondary vertex, as shown in Fig.13. This is a strong indication that, except for the unmeasured neutrino from the semileptonic $b$ quark decay, the rest of the event is well measured. This is also supported by the fact that the measured invariant mass of the two non $b$-tagged jets is $92.1 \mathrm{GeV} / \mathrm{c}^{2}$, consistent with a $Z^{0}$ boson. The measured invariant mass of the $b$-tagged jets and the missing momentum is $114.4 \mathrm{GeV} / \mathrm{c}^{2}$, which renders unlikely the $Z^{0} Z^{0}$ hypothesis.

Due to the low value of the smallest angle between 
the four jets $\left(37^{\circ}\right)$, the most likely background explanation for this event is the $b \bar{b} g g$ hypothesis. The minimum jet-jet angle for the $b \bar{b} g g$ background peaks at low values with $42 \%$ of the events having angles less than $37^{\circ}$, while $11 \%$ of the signal events have such a low angle. The two measured jet energies of the non $b$-jets, 43.5 $\mathrm{GeV}$ and $49.0 \mathrm{GeV}$, are typical, however, for the decay of a $Z^{0}$ boson produced nearly at rest.

\section{Confidence level estimation}

The mass is not the only information which allows Higgs boson production to be distinguished from background. Additional information is taken into account in the likelihood ratio $Q=L_{s+b} / L_{b}$, where $L_{b}$ is the likelihood of the background hypothesis and $L_{s+b}$ is the likelihood when a specific Higgs boson signal is added to the background. The likelihood ratio measures the compatibility of the experiment with a particular signal mass hypothesis. The likelihood ratio is traditionally shown in the form $-2 \ln Q$ because if the relationship between the likelihood ratio and the $\chi^{2}$ distributions, and also due to the fact that when the logarithm is taken individual events contribute as a sum of event weights which can be examined individually.

$$
\begin{gathered}
-2 \ln Q=2 \ln \frac{\exp ^{-(s+b)}}{\exp ^{-b}} \prod_{i=1}^{n_{o b s}} \frac{s f_{s}\left(\vec{X}_{i}\right)+b f_{b}\left(\vec{X}_{i}\right)}{b f_{b}\left(\vec{X}_{i}\right)} \\
-2 \ln Q=2 s-2 \sum_{i=1}^{n_{o b s}} \ln \left(1+s f_{s} / b f_{b}\right)
\end{gathered}
$$

here $s$ stands for the total number of signal and $b$ for background events expected, $f_{s}$ and $f_{b}$ are the probability densities that a signal or a background event will be found in a given final state with the set of values $\vec{X}_{i}$ which includes the reconstructed mass, the $b$ tagging, the jet reconstruction algorithm, the missing energy, the lepton pair selection criteria, the quality of the fit and some neural network analysis (in case it was used). Neglecting the $f_{s}$ and the $f_{b}$ terms $Q$ is simply the ratio of the Poisson probabilities to observe $n_{o b s}$ events for the signal-plus-background hypotheses and background-only hypotheses.

No correlation was found except between the neural network ALEPH analysis for the four-jets and the reconstructed Higgs mass for both the background and the signal distributions. The effect of this correlations was taken into account.

The compatibility of an experiment with a given hypothesis is determined from the expected distribution of the likelihood ratio by calculating the probability of obtaining a likelihood ratio smaller than the one observed. This probability, called the confidence level (CL), depends upon the hypothesized Higgs boson mass for both the signal-plus-background and the background-only hypotheses. If the hypothesis being tested is true, then the distribution of possible confidence levels is equally distributed between 0 and 1 , with a median value of 0.5. A signal is expected to produce an excess relative to the expected background, which would appear as a dip in the $1-C L_{b}$ distribution, where $C L_{b}$ is the confidence level for the backgroundonly hypothesis.

\section{A. Results}

Fig.14 gives the $\log$-likelihood, $-2 \ln \mathrm{Q}$, as a function of the Higgs mass. The black region shows the $1 \sigma$ limit and the gray region the $2 \sigma$ limit for the expected background. The curve (dotted) shows the expected signal+background distribution for a Higgs mass of 115 $\mathrm{GeV} / \mathrm{c}^{2}$ and the full line the results obtained from the data.

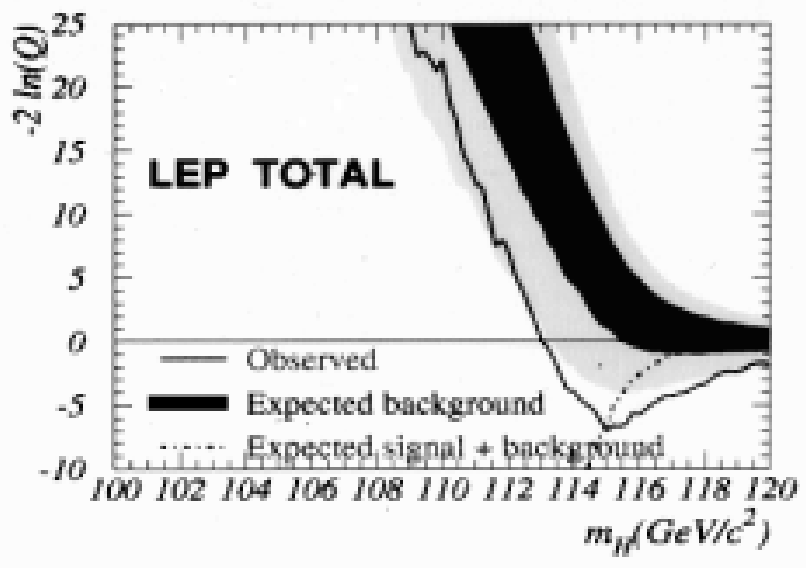

Figure 14. The log-likelihood, $-2 \ln \mathrm{Q}$, as a function of the Higgs mass. The black region shows the $1 \sigma$ limit and the gray region the $2 \sigma$ limit for the expected background. The curve (dotted) shows the expected signal+background distribution for a Higgs mass of $115 \mathrm{GeV} / \mathrm{c}^{2}$ and the full line the results obtained from the data.

Fig.15 shows the probability density as a function of log-likelihood for each experiment, separately. The vertical line shows the value of $-2 \ln Q$ found in the data, the curve drawn in a full line the expected distribution for the background and the curve in a broken line the expected distribution for the signal of a Higgs with mass $115 \mathrm{GeV} / \mathrm{c}^{2}$. As described before, ALEPH has 3 candidates, L3 has 1 candidate with a not so high probability. OPAL has shown no candidate with a high probability but combining their events they also show a displacement of the data in the direction of the expected signal+background probability distribution. DELPHI has no candidate. At the DELPHI colliding point the beams had an angle smaller than $180^{\circ}$ which brought an incertainty in the determination of the secondary vertex of the b-decays. This uncertainty was enough to discard earlier Higgs candidates found in the experiment. 


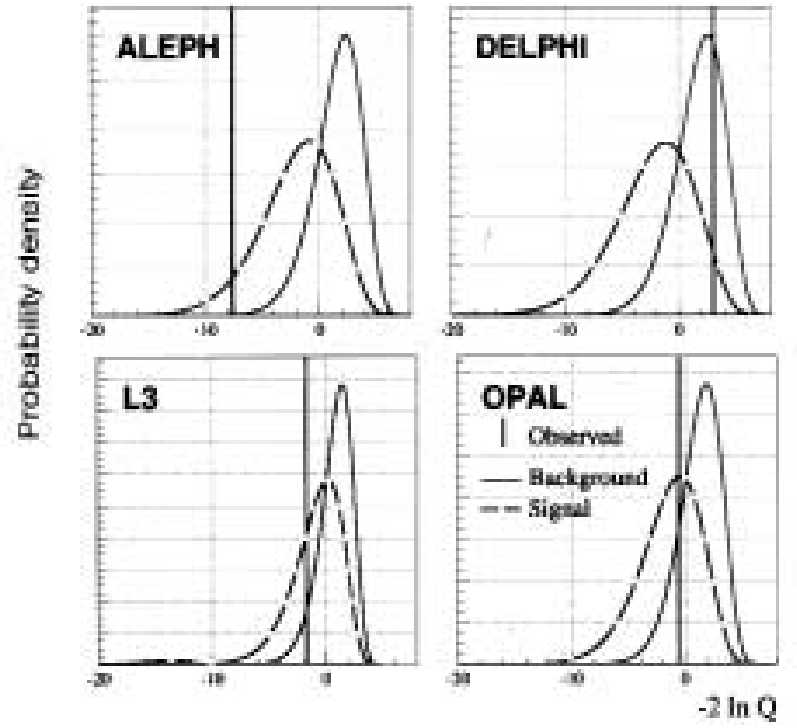

Figure 15. The probability density as a function of loglikelihood for the events found by each of the 4 experiments. The vertical line shows the value of $-2 \ln Q$ found in the data, the curve drawn in a full line the expected distribution for the background and the curve in a broken line the expected distribution for the signal of a Higgs with mass $115 \mathrm{GeV} / \mathrm{c}^{2}$.

Fig. 16 gives the same distributions for the combined results of the 4 experiments.

Fig. 17 shows the $1-C L_{b}$ distribution for the combined data from the 4 LEP experiments.

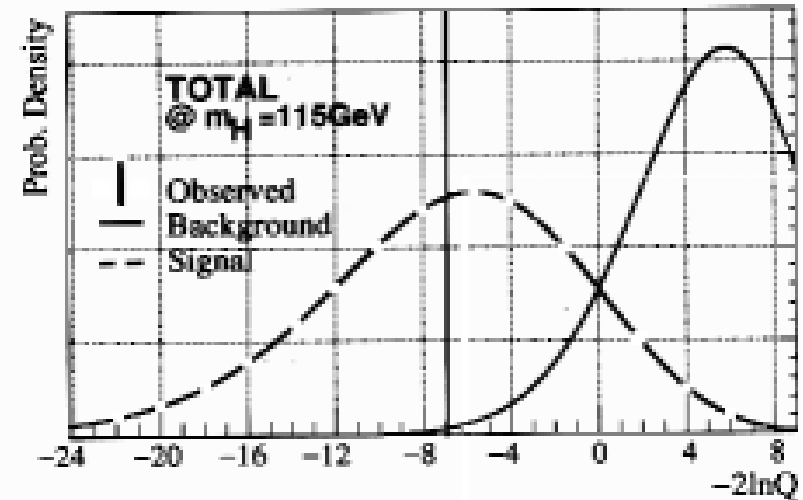

Figure 16. The probability density as a function of loglikelihood for the combined results of the 4 experiments. The vertical line shows the value of $-2 \ln Q$ found in the data, the curve drawn in a full line the expected distribution for the background and the curve in a broken line the expected distribution for the signal of a Higgs with mass $115 \mathrm{GeV} / \mathrm{c}^{2}$.

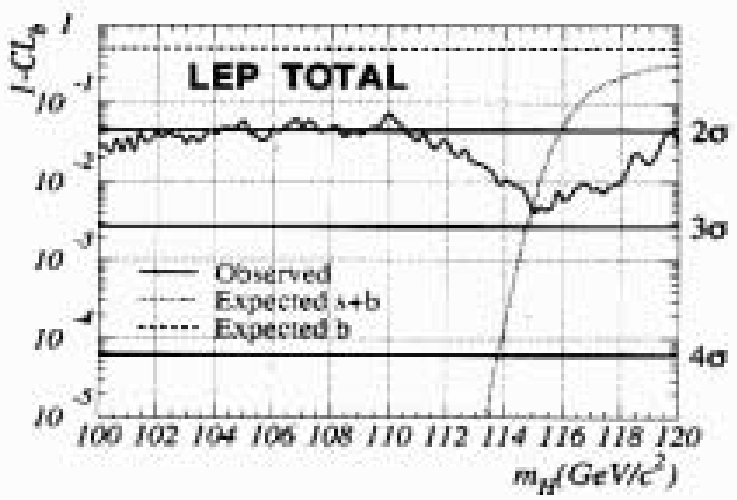

Figure 17. 1- $\mathrm{CL}_{b}$ as a function of the Higgs mass. The expected 2,3 , and $4 \sigma$ limits are drawn in the figure. The dashed line in the top shows the expected background, the curve (dotted) the expected signal+background distribution for a Higgs mass of $115 \mathrm{GeV} / \mathrm{c}^{2}$ and the full line the results obtained from the data.

It is clearly seen that an excess of events was observed, namely $2.9 \sigma$ away from the expected background. Althoug it is not enough to claim a discovery, a limit for the Higgs mass at $95 \%$ CL can be set at

$$
\mathbf{m}_{\mathbf{H}}>113.2 \mathrm{GeV} / \mathrm{c}^{2} .
$$

\section{MSSM Neutral Higgs boson}

The analysis is very similar to that done for the Standard Model Higgs, except that now we search for a pair of neutral Higgs boson, $h$ and $A$. Two channels have been studied, $e^{+} e^{-} \rightarrow h^{0} A^{0} \rightarrow b \bar{b} b \bar{b}$ and $e^{+} e^{-} \rightarrow h^{0} A^{0} \rightarrow \tau^{+} \tau^{-} b \bar{b}$, the most probable ones. The jet selection and reconstruction is the same as for the standard Higgs analysis. The final discrimination between background and signal is based on a multidimensional variable which combines eight variables including $b$-tagging, anti-QCD variables and the production angle of the Higgs boson candidates. The comparison between the data and the background simulation shows a small excess of events in the data, all with $3 b$-jets well identified but they are totally compatible with $Z^{0} Z^{0}$ events.

A tighter cut in the likelihood of the Standard Model Higgs analysis for the channel $e^{+} e^{-} \rightarrow \tau^{+} \tau^{-} q \bar{q}$ is needed in order to select the $e^{+} e^{-} \rightarrow h^{0} A^{0} \rightarrow$ $\tau^{+} \tau^{-} b \bar{b}$ candidates. No candidate was found while 0.25 are expected from Standard Model background, keeping a reasonable efficiency for the signal.

The results are combined with the same statistical method as it was for the Standard Model Higgs. In the MSSM, at tree level, the production cross-sections and the Higgs branching fractions depend on two free parameters, $\tan \beta$ and one Higgs boson mass, or, alternatively, two Higgs boson masses, in general $m_{A}$ and $m_{h}$. The properties of the MSSM Higgs bosons are modified 
by radiative corrections which introduce additional parameters: the mass of the electroweak scale, $M_{\text {susy }}$, the common $\mathrm{SU}(2)$ gaugino mass term ${ }^{1}$ at the electroweak scale, $M_{2}$, and the common squark trilinear coupling at the electroweak scale, A. The interpretation of the experimental results depends on the values assumed for these parameters and also on the order of the calculated radiative corrections[6].

The results presented here rely on leading-order two-loop calculations of the radiative corrections in the renormalization group approach[7], with recent modifications [8]. The values adopted for the parameters are: $175 \mathrm{GeV} / \mathrm{c}^{2}$ for the top mass, $1 \mathrm{TeV} / \mathrm{c}^{2}$ for $M_{\text {susy }}, 200 \mathrm{GeV} / \mathrm{c}^{2}$ for $M_{2}$ and $-200 \mathrm{GeV} / \mathrm{c}^{2}$ for $\mu$, the Higgs mixing parameter in the superpotential. For the mixing in the stop sector the $m_{h}^{\max }$ scenario and no mixing were considered. Then a scan over the MSSM parameters $\tan \beta$ and $m_{A}$, in the $m_{A}$ range of $20 \mathrm{GeV} / \mathrm{c}^{2}$ $1 \mathrm{TeV} / \mathrm{c}^{2}$, and $\tan \beta$ between 0.5 and 30 . At each point of the parameter space, the $\mathrm{hZ}$ and $\mathrm{hA}$ cross-sections and the Higgs branching fractions were computed with the HZHA program[9] (version 3).

The results translate into regions of the MSSM parameter space excluded at $95 \%$ confidence level or more. The excluded regions are presented in the $\left(m_{h}, \tan \beta\right)$ and in the $\left(m_{A}, \tan \beta\right)$ plane in Figs. 18 and 19, the first for the maximal $m_{h}$ and the second for the no mixing case. The $\left(m_{A}, m_{h}\right)$ plane is presented in Fig. 20. As ilustrated in the latter, there is a small region of the parameter space where the decay $h \rightarrow A A$ opens, in which case it suplants the $h \rightarrow b \bar{b}$ decay. This explains the unexcluded hole at $\tan \beta=0.5$ and $m_{a} \approx 40 \mathrm{GeV} / \mathrm{c}^{2}$ in the no mixing scenario. A scan for large values of $\mu$ was also done. The results, obtained by using all available LEP 2 data, are shown in the $\left(m_{h}, \tan \beta\right)$ plane and in the $\left(m_{A}, \tan \beta\right)$ plane in Fig. 21. In this case, the $\mathrm{BR}\left(h^{0} \rightarrow b \bar{b}\right) \rightarrow 0$ due to large loop corrections and the process $e^{+} e^{-} \rightarrow h^{0} A^{0}$ is suppressed.
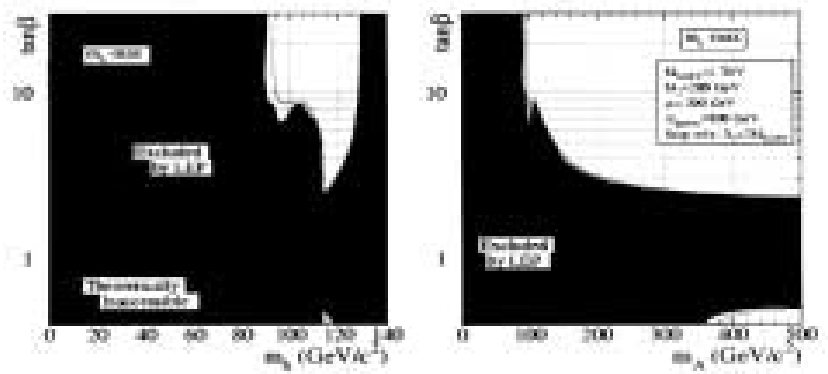

Figure 18. The excluded regions of the MSSM parameter space excluded at $95 \%$ confidence level or more for the maximal $m_{h}$ case.
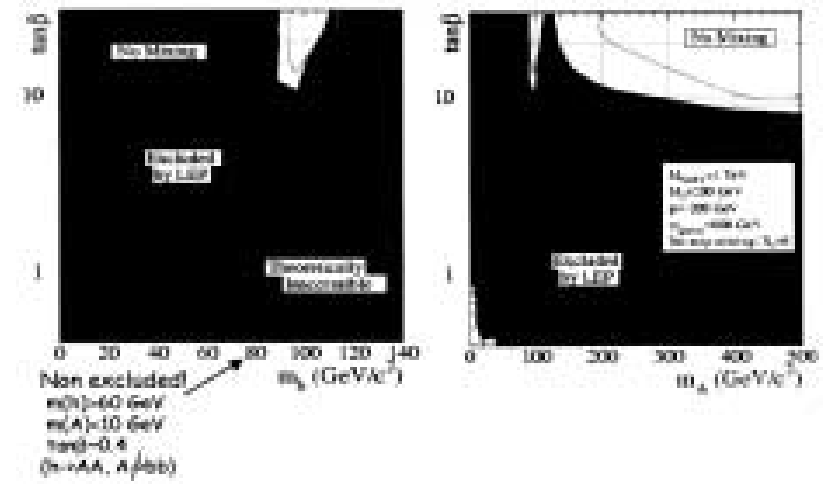

Figure 19. The excluded regions of the MSSM parameter space excluded at $95 \%$ confidence level or more for the no mixing case.
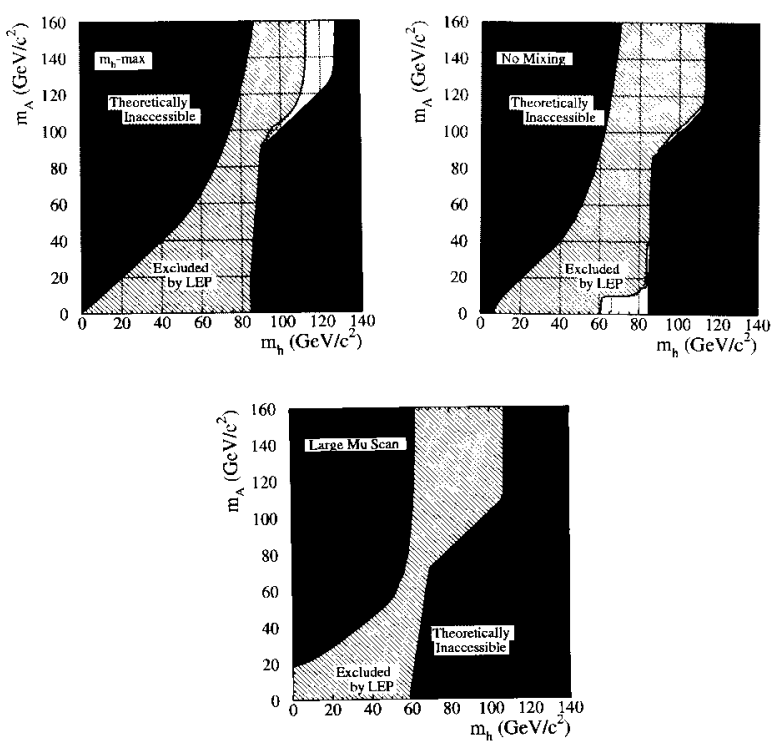

Figure 20. The excluded regions of the $m_{A}, m_{h}$ plane excluded at $95 \%$ confidence level or more.
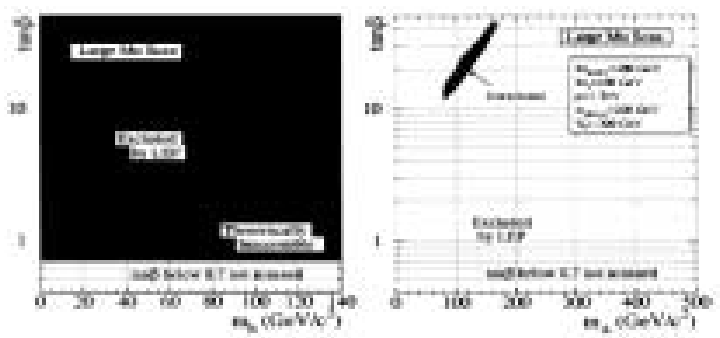

Figure 21. The excluded regions of the MSSM parameter space excluded at $95 \%$ confidence level or more for the scan over large values of $\mu$.

Finally, these results establish $95 \%$ confidence level lower limits on $m_{h}$ and $m_{A}$, for either assumption on the mixing in the stop sector and for all values of $\tan \beta$ above 0.6:

$$
m_{h}>89.9 \mathrm{GeV} / \mathrm{c}^{2} \quad m_{A}>90.5 \mathrm{GeV} / \mathrm{c}^{2}
$$

\footnotetext{
${ }^{1}$ The U(1) and SU(3) gaugino mass terms at the electroweak scale, $M_{1}$ and $M_{3}$, are assumed to be related to $M_{2}$ through the GUT relations $M_{1}=(5 / 3) \tan ^{2} \theta_{W} M_{2}$ and $M_{3}=\left(\alpha_{s} / \alpha\right) \sin ^{2} \theta_{W} M_{2}$
} 
Furthermore, there are excluded ranges in $\tan \beta$ between 0.5 and 2.3 in the no mixing case and between 0.9 and 7.7 in the $m_{h}^{\max }$ scenario as can be seen in Figs. 18 and 19.

\section{Charged Higgs bosons}

In the MSSM the $H^{ \pm}$is constrained to be heavier than the $W^{ \pm}$. The sensitivity of the current searches are limited at $m_{H}<m_{W}$ then any signal of $H^{+} H^{-}$would indicate new physics beyond the MSSM or an extreme set of parameter values.

The charged Higgs can either decay in cs quarks or $\tau \nu$. The branching ratios are unknown. Special care is needed with the $H^{+} H^{-} \rightarrow \tau \nu \tau \nu$ channel because 4 neutrinos will be present in the event. Angular cuts and a good identification for the taus were used. For the $\mathrm{H}^{+} \mathrm{H}^{-} \rightarrow$ cscs channel energy-momentum conservation and equal di-jet masses were required. For all channels, including the semi-leptonic $H^{+} H^{-} \rightarrow c s \tau \nu$ anti- $b$-tagging, WW and QCD cuts were applied taking into account the $\tau$ polarization, the acoplanarity and event shape variables, $c$ - and $s$-tagging, and the polar angles (to take into account the spin of the Higgs bosons produced).

The LEP Higgs working group has found no indication that the WW wall was broken for any branching ratio of the Higgs decay setting a mass limit at $95 \%$ confidence level

$$
\begin{aligned}
& m_{H^{ \pm}}>80.8 \mathrm{GeV} / \mathrm{c}^{2} \quad B R(\tau \nu)=0 \\
& m_{H^{ \pm}}>87.7 \mathrm{GeV} / \mathrm{c}^{2} \quad B R(\tau \nu)=1 \\
& m_{H^{ \pm}}>77.4 \mathrm{GeV} / \mathrm{c}^{2} \quad \text { any } B R(\tau \nu)
\end{aligned}
$$

Fig. 22 shows the limits obtained in the plane $\mathrm{BR}\left(H^{ \pm} \rightarrow \tau \nu\right)$ versus the charged Higgs mass.

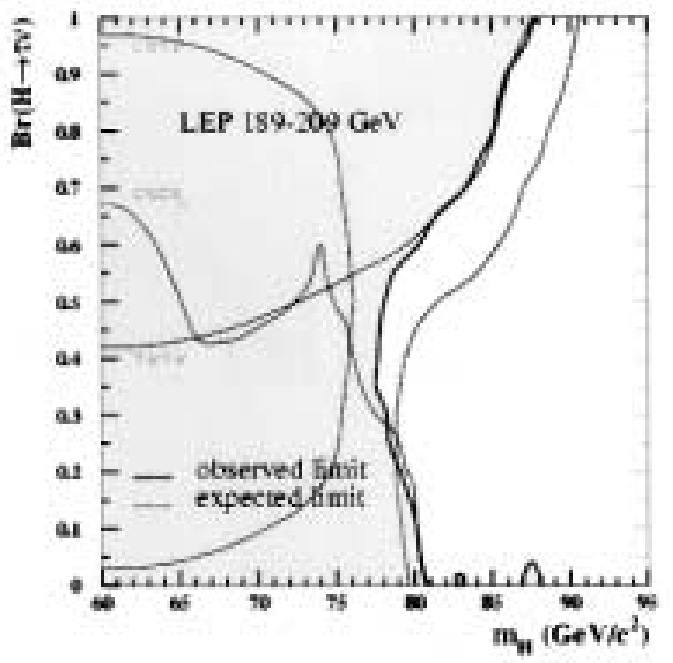

Figure 22. The upper limit for the charged Higgs mass as a function of the $\operatorname{BR}\left(H^{ \pm} \rightarrow \tau^{ \pm} \nu\right)$ at $95 \%$ confidence.

\section{Non fermionic Higgs cou- pling $h \rightarrow \gamma \gamma$}

In the Standard Model and in the Two Higgs Doublet Model we can produce the Higgs via the channels

$$
\begin{gathered}
e^{+} e^{-} \rightarrow h^{0} A^{0} \rightarrow \gamma \gamma A^{0} \rightarrow \gamma \gamma b \bar{b} \\
e^{+} e^{-} \rightarrow h^{0} A^{0} \rightarrow h^{0} h^{0} Z^{0} \rightarrow \gamma \gamma \gamma \gamma \nu \bar{\nu} \\
e^{+} e^{-} \rightarrow h^{0} A^{0} \rightarrow A^{0} A^{0} A^{0} \rightarrow b \bar{b} b \bar{b} b \bar{b} \\
e^{+} e^{-} \rightarrow h^{0} Z^{0} \rightarrow \gamma \gamma \nu \bar{\nu} \rightarrow \gamma \gamma q \bar{q}
\end{gathered}
$$

The $h^{0} \rightarrow \gamma \gamma$ channel has been searched by the 4 experiments. All the information from the electromagnetic calorimeters were used as well as the hermeticity and the $b$-tagging. The reconstructed mass for the $\gamma \gamma$ pair has shown a good agreement with the expected background. The upper limit as a function of the Higgs mass is given in Fig.23.

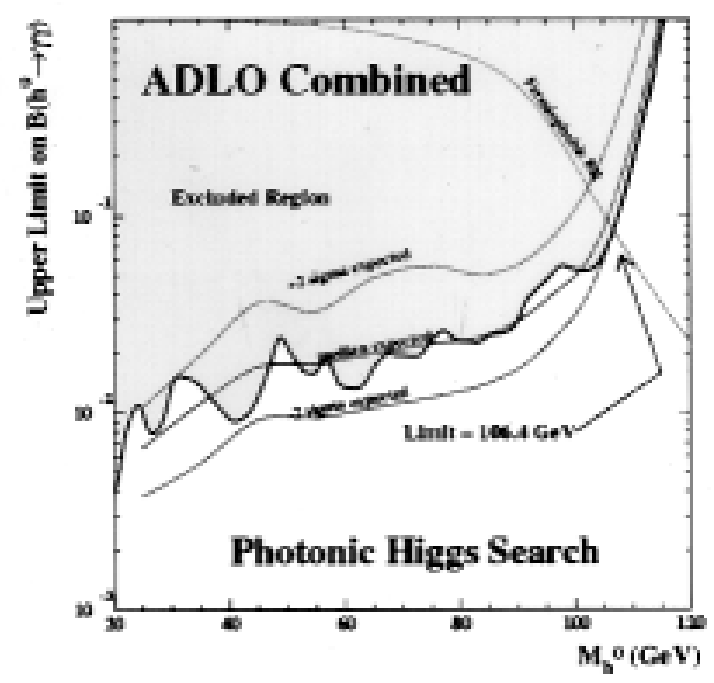

Figure 23. The upper limit as a function of the Higgs mass at $95 \%$ confidence level for the $h^{0} \rightarrow \gamma \gamma$ search.

\section{The invisible Higgs}

The Higgs boson decays into the heaviest kinematically accessible particles. In the MSSM it can decay into invisible sparticles, like the lightest neutralino $\chi^{0}$

$$
h^{0} \rightarrow \chi_{1}^{0} \chi_{1}^{0}
$$

If the $\chi^{0}$ is purely photino then this process is suppressed and

$$
h^{0} \rightarrow \chi_{2}^{0} \chi_{1}^{0} \quad ; \quad \chi_{2}^{0} \rightarrow \chi_{1}^{0} Z^{0} / \gamma^{*}
$$


The final state is then characterized by 2 leptons or 2 jets from the $Z^{0}$ plus missing energy and momentum. When the $Z^{0}$ decays into a pair of quarks, the analysis is the same as for the $H^{0} \nu \nu$ channel but here there was no need of the $b$-tag. The main backgrounds come from $e^{+} e^{-} \rightarrow q \bar{q}(\gamma), W^{ \pm} e^{\mp} \nu$ and $W^{+} W^{-}$for the case $Z^{0} \rightarrow \ell^{+} \ell^{-}$.

ALEPH, DELPHI and OPAL combined their data and found no signal putting a limit of $107.6 \mathrm{GeV} / \mathrm{c}^{2}$ for the Higgs mass at $95 \%$ confidence level.

\section{Conclusions}

LEP has had a very successful running over almost 12 years, opperating at energies much above its design value. If LEP would have reached $7 \mathrm{GeV}$ more in the centre-of-mass energy than what it has delivered, the MSSM would have been covered completely, although strong constraints have been put (also from the combined analysis of the 4 experiments):

$$
\begin{gathered}
m\left(h^{0}\right), m\left(A^{0}\right)>90.5 \mathrm{GeV} / c^{2} \\
0.9>\tan \beta>2.3 \\
m\left(H^{ \pm}\right)>77.4 \mathrm{GeV} / c^{2} \\
m(L S P)>48 \mathrm{GeV} / c^{2} \\
m\left(\chi^{ \pm}\right)>104 \mathrm{GeV}
\end{gathered}
$$

The results presented here were shown at the LEPC meeting at CERN on November 3, 2000 and also published by the 4 experiments, as given in the references. More data, or results, from other experiments, rather than the LEP ones, will be needed to determine wether the observations reported here are the result of a statistical fluctuation or the first sign of direct production of the Higgs boson.

\section{References}

[1] ALEPH Collaboration, D. Abbaneo et al., Nucl. Instr. and Meth. A294, 121 (1990).
ALEPH Collaboration, D. Abbaneo et al., Nucl. Instr. and Meth. A360, 481 (1995).

DELPHI Collaboration, P. Abreu et al., Nucl. Instr. and Meth. A378, 57 (1996).

DELPHI Sillicon Tracker Group, P. Choudoba et al., Nucl. Instr. and Meth. A412, 304 (1998).

L3 Collaboration, B. Adeva et al., Nucl. Instr. and Meth. A289, 35 (1990).

OPAL Collaboration, K. Ahmet et al., Nucl. Instr. and Meth. A305, 275 (1991).

OPAL Collaboration, S. Anderson et al., Nucl. Instr. and Meth. A403, 326 (1998).

OPAL Collaboration, B.E. Anderson et al., IEEE Trans. on Nucl. Science 41, 845 (1994).

OPAL Collaboration, G. Aguillion et al., Nucl. Instr. and Meth. A417, 266 (1998).

[2] http://delphiwww.cern.ch/offline/physics_ links/lepc.html http://lephiggs.web.cern.ch/LEPHIGGS

[3] ALEPH Collaboration, CERN - EP/2000 - 138, R. Barate et al., Phys. Lett. B495, 1 (2000).

DELPHI Collaboration, CERN - EP/2000 - 285, P. Abreu et al., Phys. Lett. B499, 23 (2001).

L3 Collaboration, CERN-EP/2000-xxx, M. Acciarri et al. Phys. Lett. B495, 18 (2000).

OPAL Collaboration, CERN-EP/2000-156, G. Abbiendi et al., Phys. Lett. B499, 38 (2001).

http://r.home.cern.ch/r/rembser/www/lepc_journ.html

[4] L3 Collaboration, CERN - EP/2000 - xxx, M. Acciarri et al. Phys. Lett. B496, 34 (2000).

[5] Proceedings of the International Conference for High Energy Physics - ICHEP200 - Osaka, Japan, to be published.

[6] Luc Pape, Proceedings of the X Swieca Summer School - Particles and Fields, World Scientific (2000).

[7] M. Carena, M. Quiros and C. Wagner, Nucl. Phys. B461, 407 (1996).

[8] S. Heinemeyer, W. Hollik and C. Weiglein, JHEP 6 (2000) 9.

M. Carena, S. Heinemeyer, C.E.M. Wagner and C. Weiglein, CERN-TH/99-374.

[9] http://alephwww.cern.ch/ janot/Generators.html 